\title{
TEMA -2017: Osteomielitis, miembro inferior y PMMA.
}
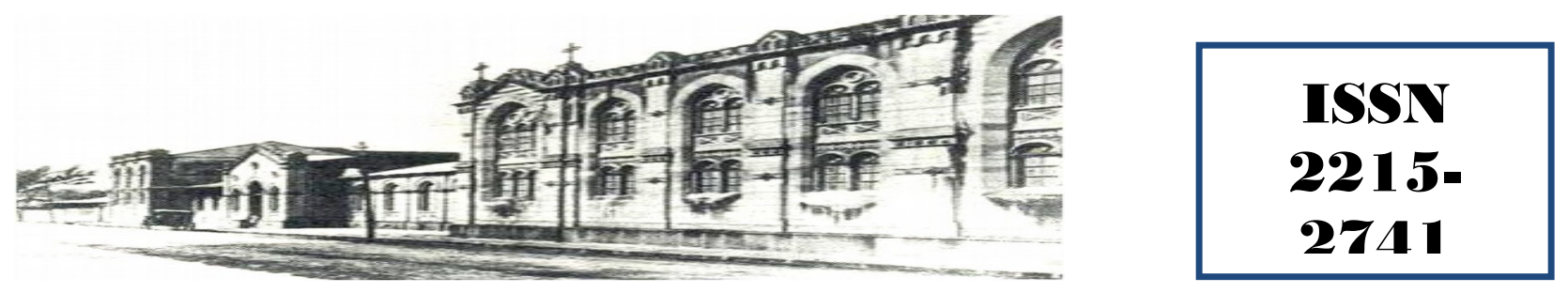

Haspital San quan de Dias, San Jasé, Casta Rica. Fundada en 1845

Recibido:

$28 / 10 / 2017$

Aceptado:

$15 / 12 / 2017$

Adriana Paola Arroyo López ${ }^{1}$

Hebel José Solano Múñoz ${ }^{2}$

Víctor Manuel Rojas Murillo ${ }^{3}$

${ }^{1}$ Médico general, Universidad Latina de Costa Rica, Caja Costarricense del Seguro Social. Correo electrónico adrial.arroyo@gmail.com

${ }^{2}$ Médico Residente, Cirugía General Hospital Max Peralta, Universidad Latina Costa Rica. Correo electrónico: hsmc1089@gmail.com

${ }^{3}$ Médico Asistente Cirugía General, Universidad de Costa Rica, Hospital Max Peralta. Correo electrónico: dr.victor.rojas@gmail.com

\section{RESUMEN}

La osteomielitis es una enfermedad que presenta un crecimiento exponencial durante los últimos años; en relación con el incremento de traumas y los incontables procedimientos quirúrgicos invasivos que se realizan en la actualidad, como los reemplazos articulares. Aunado a esto, la presencia de ciertas patologías como Diabetes Mellitus, que provoca un aumento significativo de las infecciones óseas, culminando en un proceso de tratamiento que suele ser largo y reincidente. Cabe destacar que la osteomielitis no discrimina edad, género, y se presenta con frecuencia en la población infantil.

Este artículo proporciona al lector información de las técnicas más recientes en el abordaje y tratamiento de la osteomielitis, partiendo de lineamientos integrales, para consolidar un tratamiento con preservación de la articulación. La base de este artículo es el tratamiento quirúrgico, haciendo hincapié en el diagnóstico.

\section{PALABRAS CLAVE}

Osteomielitis. Clasificación de osteomielitis abordaje quirúrgico de osteomielitis. Osteomielitis aguda. Osteomielitis crónica. Osteomielitis en miembro inferior. Biofilm. VAC. PMMA.

\section{ABSTRACT}


Osteomyelitis is an infectocontagious disease, that recently has risen in its incidence due to an elevated rate of trauma and orthopedic surgeries such as and specially joint replacement. Today there's an abundance of surgical and medical approaches, which have allowed to control this elevated incidence. This article has the objective of providing the reader with knowledge and understanding of the most recent and evidenced based medical treatment but predominantly surgical options to ensure the best outcome in terms of survival and morbidity for the patients suffering from the disease.

\section{KEY WORDS}

Osteomyelitis. Surgical approach osteomyelitis. Osteomyelitis and classification. Acute osteomyelitis. Chronic osteomyelitis. Diabetic foot. Lower limbs. Biofilm. VAC. PMMA.

\section{INTRODUCCIÓN}

El tratamiento refractario de las infecciones agudas, es una de las principales causas de osteomielitis crónica. En cirugías electivas secundarias a trauma, la tasa de incidencia ronda entre $1 \%$ a un $5 \%{ }^{(1)}$. Uno de los pilares fundamentales en el manejo de la osteomielitis es precisamente el diagnóstico temprano y específico del agente patógeno causal, que es elemental para dar inicio a la terapia antibiótica. El principal causante de la infección de hueso es el Sthaphylococcus aureus; las infecciones con una fractura expuesta o un reemplazo articular asociado deben ser tratados inmediatamente con combinación de terapia antibiótica y quirúrgica.

\section{DISCUSIÓN}

\section{Definición y Fisiopatología}

La osteomielitis es un proceso infeccioso e inflamatorio que culmina en necrosis y destrucción del tejido óseo. En los países industrializados los eventos traumáticos y los pos operatorios son la forma más importante de las infecciones óseas. Aproximadamente un 10-30\% de estas infecciones progresan a la cronicidad $^{(1)}$

Para adentrarse en el mecanismo fisiopatológico, es necesario definir el concepto de biofilm o biopelícula: "grupo de bacterias sésiles que se en- cuentran incrustadas en una matriz polimérica extracelular o glicocálix", estas bacterias cuentan con la característica de ser más tolerante a la mayoría de antimicrobianos y a la respuesta de defensa del huésped ${ }^{(1)}$.

Está bacteria se caracterizó porque se adhiere irreversiblemente a un sustrato o interfase, o bien unas con otras, encerradas en una matriz polimérica extracelular que ellas mismas producen y exhiben un fenotipo alterado en relación con la tasa de crecimiento y transcripción génica ${ }^{(2)}$. El biofilm se encuentra compuesto en un $97 \%$ de agua, pero además de esto, la matriz presenta exopolisácaridos, los que constituyen su componente fundamental y son producidos por sus mismos microrganismos integrantes ${ }^{(3)}$.

Como se resume en la ilustración, figura 1, existe inflamación del hueso y matriz ósea, por la presencia de una bacteria en el hueso, provocando de manera secundaria la elevación de leucocitos que contribuyen precisamente a la necrosis y a destrucción del hueso trabecular y matriz ósea.

Cuando la infección se disemina al canal medular, aumenta la presión, ocasionando la extensión a la corteza ósea a través de los canales de Haversianos y de Volkman, con la consecuente diseminación en el espacio subperióstico y finalmente en periostio y tejidos adyacentes. Los canales vasculares sufren compresión y son obliterados por el proceso inflamatorio, dando como resultado un proceso isquémico. Segmentos del hueso quedan desprovistos de aporte sanguíneo y se produce necrosis del mismo.

Estos segmentos óseos se pueden separar y originar secuestros, (segmento necrótico del hueso que es separado consecuente al tejido de granulación). Posterior a esta etapa, inicia un proceso de actividad osteoclástica aumentada, (relacionada con infarto del área), pérdida de hueso y osteoporosis.

\section{Patogénesis}

El S. aureus tiene la capacidad para adherirse a través de adhesinas, dicha habilidad favorece a la colonización de los tejidos del huésped y son capaces de formar biofilm ${ }^{(4)}$.

Estadío clínico de la osteomielitis: El sistema más común utilizado para la práctica clínica de 
osteomielitis es la clasificación de Cierny-Mader y Waldvogel ${ }^{(5)}$.

Las limitaciones que presenta este sistema hacen que sea preferible utilizar el sistema de CiernyMader, basada en hallazgos clínicos, anatómicos y radiológicos. Este divide la osteomielitis en cuatro estadios.

Dentro de esta misma clasificación se añade una segunda dimensión, donde el huésped juega un papel fundamental.

Está clasificación presenta la limitación de: no contempla la duración en la que la infección ha persistido, si hay relación con dispositivos médicos o el rango de edad del paciente ${ }^{(5)}$.

Este tipo de clasificación destaca que dentro los tipos de osteomielitis también toma en cuenta la osteomielitis vertebral. Asociada a la siembra de un foco hematógeno en el espacio en un disco intervertebral adyacente, de un foco distante. Por lo general el diagnóstico se ve atrasado porque se puede confundir con un proceso degenerativo.

Se diagnostica por el dolor de espalda recalcitrante que no responde a medidas conservadoras y marcadores inflamatorios elevados con o sin fiebre. Las radiografías simples de columna no son sensibles para el diagnóstico precoz. El agente causal es monomicrobiano: S. aureus; por lo general resuelve en 6 semanas, sin embargo algunos pacientes necesitan desbridación o estabilización espinal, posterior a terapia antibiótica ${ }^{(6)}$.

En el caso de la osteomielitis hematógena, se presenta en poblaciones más jóvenes, y las principales causas en niños se producen por: Inoculación directa, reducción quirúrgica, fijación interna y contaminación nosocomial. En estos casos el patógeno causal está directamente asociado con la edad:

Si el paciente tiene antecedente de varicela previa se debe de tener en cuenta el S. pyogenes. La enfermedad de las células falciformes indica Salmonella y S. aureus ${ }^{(7)}$.

\section{Tratamiento médico de osteomieli- tis}

El primer componente de tratamiento consiste en la optimización médica del paciente para promover la integridad del hueso y la cicatrización de heridas.

La duración óptima de la terapia es alrededor de 4 a 6 semanas. Este tipo de tratamiento es resolutivo en los casos de osteomielitis aguda, en el caso de la progresión a osteomielitis crónica es necesario implementar técnicas quirúrgicas.

La complementación de tratamiento intravenoso u oral, con el tratamiento quirúrgico, es de suma importancia para el éxito de la terapia. Para uso parenteral se utiliza betalactámicos ${ }^{(8)}$.

Por ejemplo, en el caso de S. aureus penicilino resistente se suele utilizar penicilina benzatínica en dosis diarias de 12 a 20 millones de unidades, para los S. aureus meticilino resistente se puede utilizar nafcilina 1 o 2 g cada 4-6 h o cefazolina $2 \mathrm{~g}$ cada 8 horas. En el caso de Streptococcus, ya sea del grupo A o beta hemolítico o S. pneumoniae, se puede utilizar vancomicina $1 \mathrm{~g}$ cada $12 \mathrm{~h}$ o de igual forma penicilina benzatínica en dosis diarias de 12 a 20 millones de unidades.

Para bacilos enterococos o gram negativos se utiliza quinolonas, por ejemplo: ciprofloxacina en dosis de 400 a $750 \mathrm{mg}$ cada $12 \mathrm{~h}$. En el caso de Serratia sp., P. Aeruginosa, piperacilina de 2 a 4 $\mathrm{g}$ cada 4 horas y aminoglucósidos. Para agentes anaerobios clindamicina $600 \mathrm{mg}$ cada $6 \mathrm{~h}$ y en infecciones mixtas ampicilina-sulbactam 2-3g cada 6-8h.

Con respecto a los agentes orales hay ciertos estudios que demuestran que estos pueden superar las concentraciones mínimas inhibitorias de bacterias, en particular las fluoroquinolonas, linezolid y $\mathrm{TMP}_{\mathrm{SMX}}{ }^{(8)}$. Otros tratamientos que se pueden utilizar son la doxiciclina y la clindamicina.

En el caso del fallo con estas terapias se recomienda utilizar daptomicina. 


\section{Tratamiento Quirúrgico}

Dentro de las técnicas de tratamiento para las secciones de hueso infectadas, con el fin de conseguir la estabilidad axial, el cirujano tiene dos opciones $^{(9)}$ :

1. Resección del hueso con la subsecuente desestabilización de la extremidad.

2. Dejar tejido dañado tratando de erradicar o contener la infección.

La corrección de factores modificables como: mal control glicémico, uso del tabaco, desnutrición, alteraciones endocrinas y la insuficiencia vascular del miembro afectado, son vitales para evitar la mala cicatrización posoperatoria y disminuir el riesgo de recurrencia. El manejo de la osteomielitis crónica ha cambiado drásticamente durante los últimos 25 años con el uso de colgajos e injertos óseos ${ }^{(10)}$.

\section{Opciones quirúrgicas}

\section{$\boldsymbol{P P M A}$}

El PPMA (Polimetil metacrilato) es un polvo que mezclado con antibiótico, (antibiótico en presentación de polvo), es polimerizado, esto para uso ortopédico $^{(11)}$. PMMA, además de estar compuesto por polvo en forma de polímero, está formado por un monómero líquido, este monómero liquido por lo general se suministra en relación $2: 1^{(12)}$. La combinación del polvo y del líquido produce una reacción exotérmica.

PMMA tiene diferentes usos: es utilizado para rellenar espacios dejados por materiales protésicos en extremidades. En infecciones como osteomielitis, mezclado en forma de polvo con antibióticos, en los que destaca aminoglúcosidos: tobramicina; (es popular por su presentación en polvo y presenta un amplio espectro que incluye cobertura contra pseudomona). También se encuentra en uso la gentamicina o la vancomici$\mathrm{na}^{(12)}$.
El monómero de metilmetacrilato, el componente básico del PMMA, contiene cadenas dobles de carbón que reaccionan con radicales libres. El monómero es libre de interactuar con otras moléculas de otros monómeros produciendo un crecimiento de la cadena de polímeros. El polvo inicia la polimerización y crea una masa maleable ${ }^{(12)}$.

\section{Empleo de la técnica con PPMA}

Este procedimiento presenta dos etapas. La primera etapa de la técnica consiste en una desbridación del tejido contaminado y del tejido fibroso. El tratamiento se basa en: irrigación, desbridación y obliteración del espacio muerto. La reconstrucción del hueso únicamente será posible si existe la completa erradicación de la infección ${ }^{(13)}$. La mayor parte de la desbridación es determinada por las resonancias magnéticas que se recomiendan hacer preoperatorias.

La desbridación del hueso avascular es llevada a cabo en el punto donde haya sangrado, lo que se le conoce como el signo de paprika. Usualmente se llega a este nivel usando una broca de $2.5 \mathrm{~mm}$ de diámetro, una vez alcanzado el punto, se lleva a cabo una corticotomía con una broca y un osteomo, evitando que se comprometan los márgenes del hueso que se encuentran íntegros ${ }^{(13)}$.

Se puede colocar un fijador externo de manera temporal para mantener la longitud y la rotación antes del desbridamiento, a través de la irrigación del hueso y del tejido circundante. La escisión de las fístulas y tejidos blandos afectados completa el proceso de desbridamiento. Solo alcanzando la adecuada desbridación y fijación de la extremidad se puede iniciar a insertar el espaciador PMMA.

En la parte final de esta primera fase se administran antibióticos intravenosos por un lapso de 6-8 semanas, y se cubre de manera temporal la herida con un VAC o con colgajos libres o rotatorios y autólogos ${ }^{(14)}$. Los injertos autólogos son injertos de elección. Por lo general los injertos de hueso utilizados en fracturas óseas son: hueso 
autólogo esponjoso, cortical autólogo, corticoesponjoso vascularizado.

Los injertos de hueso autólogo esponjoso son el tratamiento de mayor elección porque favorecen a la osteoinducción, osteoinducción y osteogéne$\operatorname{sis}^{(14)}$.

El dispositivo asistido por vacío de cierre (VAC), utiliza la presión atmosférica negativa continua, por lo general $125 \mathrm{mmHg}$, que se aplica a través de un apósito de espuma de células sellada, esto sobre una herida para disminuir el edema, aumentar rápidamente la cantidad de tejido de granulación y reducir el tamaño de la herida ${ }^{(15)}$.

Actualmente en el manejo de heridas traumáticas, además del empleo del VAC, existen otras técnicas utilizadas como: Epigard, el cual es un apósito de dos capas, que simula piel humana y también se encuentra la capa superficial de teflón, fabricado con poliuretano, una capa permeable al aire, pero que evita la penetración de bacterias en el interior de la herida ${ }^{(16)}$. Actualmente el más empleado es el VAC debido a que estudios demuestran que favorece a la vascularización, angiogénesis y finalmente promueve el proceso de curación.

Otros métodos empleados son el de vidrio bioactivo S53P4; cuando el vidrio bioactivo se implanta en un defecto óseo séptico se intercambia el medio alcalino de la superficie del vidrio con el hidronio en el microambiente circundante, lo que aumenta el $\mathrm{pH}$ local.

La liberación de iones de la superficie del vidrio ocasiona un incremento de la presión osmótica a nivel local, y una capa de gel de sílice se forma cerca de la superficie del vidrio en los que, los precipitados de fosfato de calcio amorfo se van a cristalizar en hidroxiapatita natural; la misma provoca un efecto osteoestimulante mediante la activación de células osteogénicas, siendo esta de las principales ventajas de este tipo de injerto, sobre el injerto autólogo, la capacidad excelente de curación que presenta el hueso aunado a una protección contra la colonización y adhesión bacteriana en su propia superficie ${ }^{(14,17)}$.

El vidrio bioactivo S53P4 ofrece una solución de un único paso, eliminando las cirugías adicionales que se realizan en los procedimientos con PMMA, sin embargo se requieren muchos más estudios para poder reemplazar las cadenas PMMA por el vidrio bioactivo.

La segunda fase del tratamiento se lleva a cabo aproximadamente 6-8 semanas cuando se ha alcanzado por completo el proceso de recuperación del tejido blando, el espaciador es removido, pero la membrana inducida por el cemento es dejada en el lugar ${ }^{(18)}$.

\section{Técnica de bead-pouch}

Consiste en una cobertura de antibiótico cargado de polimetil mecrilato, en la que se coloca en la herida, recubierto con una membrana porosa ${ }^{(19)}$. Está membrana porosa promueve la vascularización y la corticalización del hueso esponjoso ${ }^{(18,19)}$. Para alcanzar la elución deseable y niveles terapéuticos sostenidos, tiene una presentación de cadenas o bien prótesis como espaciadores para rellenar el tejido blando y defectos óseos.

En el caso de los espaciadores, los cuales pueden ser estáticos o móviles, han demostrado ser eficaces en el tratamiento de las infecciones prostéticas de las articulaciones. Existen espaciadores creados para rodilla con componentes femorales que han alcanzado excelentes resultados. En el caso de los espaciadores móviles de cadera han permitido disminuir el tiempo de hospitalización y han demostrado una reducción en la tasa de reimplantación y dislocación ${ }^{(20)}$.

En la actualidad, aunque no se emplea, se han realizado estudios con gentamicina líquida, que ha demostrado tener una alta efectividad y no se ha correlacionado con nefrotoxicidad ${ }^{(21)}$. Un estudio preliminar que se llevó a cabo in vitro demostró una potente actividad bactericida en la gentamicina líquida empleada en el cemento ${ }^{(22)}$. 
Por otra parte, las cadenas de PMMA, no solo se impregnan con gentamicina, también utilizan tobramicina y vancomicina. Las cadenas que se encuentran disponibles en el mercado tienen un diámetro de 7-10 $\mathrm{mm}$. Una cadena de $10 \mathrm{~mm}$ de gentamicina contiene $4.5 \mathrm{mg}$ de antibiótico. Usualmente se llegan a utilizar hasta 30 cadenas. El pico más alto de liberación de antibiótico se alcanza durante las primeras 48h, sin embargo, hay una liberación continua durante semanas, estudios en animales demuestran que la liberación se puede mantener hasta por 6 meses. Si se utiliza vancomicina se emplean $4 \mathrm{~g}$ por $40 \mathrm{~g}$ de cemento y también se puede emplear ácido fusídico $3 \mathrm{~g}^{(23)}$.

Una vez realizada la desbridación y lavado de alta presión con solución fisiológica, se coloca la cadena de antibióticos en la herida. La herida se cubre con bálsamo de Friar rodeado la piel para adherir la cobertura porosa de polietileno, obteniendo un sello impermeable de la herida, se coloca un catéter Jackson-Pratt de $10 \mathrm{~mm}$ para el lí-

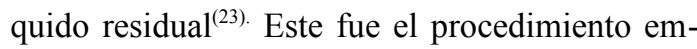
pleado en un estudio que se realizó de 1999 al 2006 donde se sometieron a estudio 84 pacientes tratados por osteomielitis pos traumática en el segmento distal del tobillo y pie.

La segunda etapa del tratamiento es llevada a cabo 6-8 semanas finalizada la primera etapa, permite la epitelización del colgajo de músculo, la revascularización del tejido viable, la formación de la membrana perióstica auto inducida y el tratamiento de cualquier infección remanente con antibióticos locales o sistémicos. Se realiza una abertura quirúrgica del defecto para remover el espaciador de cemento, con una incisión única central a través de la membrana perióstica.

La cavidad perióstica debe ser rellenada con un injerto de hueso, cuya selección es basada en la preferencia del cirujano y depende del volumen que se requiera para rellenar el vacío. La membrana es entonces, cuidadosamente cerrada para crear espacio para el injerto y los tejidos blandos restantes se cierran en capas. Se ha demostrado que no existe ningún beneficio en realizar cultivos peri o intra operatorios, ya que resultan ineficaces en la predicción de la infección o identificación del agente.

En un estudio realizado: Patzakis y Wilkins ${ }^{(15,23)}$, se demostró el significante aumento de infecciones con respecto a la administración de la primera dosis de antibiótico: profilaxis en un tiempo menor tres horas $4.7 \%$ riesgo de infección, mayor a tres horas incrementa a un $7.4 \%$. En otros estudios en animales, se planteó que una buena irrigación disminuye el riesgo de desarrollar infecciones, según la clasificación de Gustilo y Andres, para las fracturas de tipo I se recomiendan 3L de irrigación fisiológica, y 9L de irrigación para fracturas tipo III, esto asociado con sustancias antisépticas como: povidona yodada, la solución Dakin y clorhexidina ${ }^{(15,23)}$. La osteomielitis postraumática o posoperatoria es un subtipo de osteomielitis con una prevalencia del $80 \%{ }^{(24)}$.

Por lo general el fallo de los procedimientos ocurre cuando hay fallo en la antibioticoterapia o bien se realizan malas desbridaciones. En el caso por ejemplo de los pacientes con pie diabético, suelen presentar alteraciones infecciosas secundarias a ulceraciones ${ }^{(25)}$. Los pacientes diabéticos tienen una incidencia del 12 al 15\% a lo largo de la vida en desarrollar úlceras y un riesgo de al menos 15 veces más de amputaciones en pie ${ }^{(26)}$.

Las fracturas expuestas son secundarias, por lo general a traumas de alto impacto; está exposición del hueso y tejido provoca un incremento considerable en el rango de infección y de complicaciones ${ }^{(27)}$. La clasificación Gustilo-Anderson pese a las limitaciones, es pronostica para determinar el desarrollo de infecciones en ortopedia.

\section{Clasificación de Gustilo-Anderson}

Tipo I: Fractura simple con una herida de menos de $1 \mathrm{~cm}$ de longitud, con contaminación mínima o mínimo compromiso muscular. (0-2\%) 
Tipo II: Indica laceración de más de un centímetro de longitud con daño moderado del tejido. (2$10 \%)$

Tipo III: el tipo III se subdivide en A y B. (10$50 \%)$

Para la clasificación de las heridas abiertas se recomienda realizarlas en sala de operaciones, posterior a la exploración y desbridamiento de la herida.

\section{PMMA, indicaciones, contraindicaciones ${ }^{(28)}$ :}

Indicaciones:

- Prevención de infecciones en fracturas abiertas.

- Tratamiento en la estabilización del hueso, por ejemplo, en las osteomielitis agudas y crónicas.

- Tratamiento de artroplastias infectadas.

- Manejo del espacio muerto en pacientes con lesiones extensas de tejido blando

- Reemplazos articulares infectados

Contraindicaciones:

- Hipersensibilidad a antibióticos específicos.

- Heridas pequeñas para las cuales no son necesarias

- Extremidades no viables.

\section{Diagnóstico}

La osteomielitis es primariamente de diagnóstico clínico. El método diagnóstico de elección es el cultivo de la muestra y la identificación del agente. Se pueden realizar estudios de laboratorio tal como: VES y PCR.

La proteína $\mathrm{C}$ reactiva es efectiva en la valoración control de los pacientes que se encuentran en tratamiento. Además de los estudios imageno- lógicos ya conocidos, se emplean nuevas tecnologías como la gammagrafía ósea: difosfonato de metileno, que se une a los sitios donde la actividad metabólica ósea está aumentada ${ }^{(4,28)}$.

una nueva tecnología está ganando importante relevancia: reacción en cadena de la polimerasa con electrospray espectrometría de ionización de masas (PCR/ESI-MS), emplea un único ensayo que amplifica y hace más precisa la identificación de muchas bacterias aisladas. Este sistema amplifica la mayoría de especies dentro de una muestra, determinando la masa molecular, y esto a su vez, permite una derivación inequívoca de las composiciones base, que se introducen en una base de datos para la identificación del organis$\mathrm{mo}^{(29)}$.

\section{Radiografias convencionales}

Son parte de la evaluación inicial en todo paciente que se sospeche osteomielitis. En infecciones piógenas el primer cambio que se produce en hueso, y que se puede ver reflejado en la radiografía, ocurre posterior a 2 o 3 semanas de iniciado el proceso infeccioso. Por lo general para ver cambios en niños se deben esperar de 5-7 días en y de 10-14 días en adultos ${ }^{(30)}$.

Dentro de los cambios radiográficos que se pueden encontrar están: Engrosamiento del periostio, lesiones líticas, pérdida de la estructura molecular y aposición de hueso nuevo ${ }^{(28,30)}$. Por otra parte en el caso de desarrollar osteomielitis crónica se puede ver en la radiografía: hueso necrótico, que se forma en un promedio de 10 días, periostitis, hueso nuevo que se encuentra recubriendo hueso necrótico, fístulas secundarias al absceso subperióstico con levantamiento del periostio ${ }^{(30)}$.

\section{Ultrasonografía}

Limitado para el diagnóstico y manejo de osteomielitis. Se utiliza principalmente para la valoración del tejido subcutáneo ${ }^{(31)}$.

TAC 
Es mucho más accesible y tiene menor costo en comparación con la RMN. Muestra claramente la relación anatómica entre las áreas de infección y estructuras importantes. Es buena para la identificación de cambios óseos como destrucción de la cortical, reacciones periósticas y la identificación de secuestro, que aparece como un anillo reluciente. Su principal función es la identificación del secuestro óseo. Sin embargo se prefiere la RMN debido a la disminución de contraste de los tejidos blandos así como la exposición a la radiación ionizante ${ }^{(30,31)}$.

\section{$R M N$}

Provee mejor información que las otras modalidades de imagen. Puede detectar la afección con 3-5 días una vez iniciado el proceso infeccioso. La sensibilidad y la especificidad es de aproximadamente un $90 \%{ }^{(32)}$.

\section{Medicina Nuclear}

Pueden detectar la afección entre 10-14 días una vez iniciado el proceso $^{(32)}$.

En los casos secundarios a neoplasias (la mayoría), los síntomas son complejos debido a que el tumor causal puede afectar a otras estructuras; es especialmente importante estudiar la columna

\section{CONCLUSIONES}

La osteomielitis es una afección altamente común en los sistemas intrahospitalarios, sea por causas traumáticas o quirúrgicas, los avances de la ciencia han permitido desarrollar diferentes métodos que han favorecido a un mejor manejo y resolución de dicha patología.

A pesar de que queda mucho por estudiar e investigar, los hallazgos y las técnicas de hoy en día son tremendamente favorecedores, y permiten al paciente retomar su vida cotidiana con mucha practicidad. Con la utilización de las cadenas de antibióticos, se ha logrado disminuir la nefro- totoxicidad y efectos secundarios de terapias sistémicas, además se ha logrado un manejo localizado y efectivo.

\section{BIBLIOGRAFÍA}

1. Walter G Kemmerer M Kappler C Hoffmann R. Treatment algorithms for chronic osteomyelitis. Dtsch Arztebl Int. 2012 Apr;109(14):257-264. doi: 10.3238/arztebl.2012.0257. Epub 2012 Apr 6.

2. Donlan RM. Biofilms: Microbial life on surfaces. Emerg Infect Dis 2002; 8 (9): 881-890. DOI: $10.3201 /$ eid0809.020063

3. Julio Nazar C. Bacterial biofilms. Rev. Otorrinolaringol. Cir. Cabeza Cuello 2007; 67: 61-72. doi.org/10.4067/S0718$\underline{48162007000100011 .}$.

4. Lew DP Waldvogel FA. Osteomyelitis. Lancet. 2004 Jul 24-30;364(9431):369379. DOI: $10.1016 / \mathrm{S} 0140-$ $\underline{6736(04) 16727-5}$

5. Rao N Ziran BH Lipsky BA. Treating osteomyelitis: antibiotics and surgery. Plast Reconstr Surg. 2011 Jan;127 Suppl 1:177S-187S. doi: 10.1097/PRS.0b013e3182001fof.

6. Elie F. Berbari Souha S. Kanj Todd J. Kowalski Rabih O. Darouiche Andreas F. Widmer Steven K. Schmitt Edward F. Hendershot et al. Infectious Diseases Society of America (IDSA) Clinical Practice Guidelines for the Diagnosis and Treatment of Native Vertebral Osteomyelitis in Adults . Clin Infect Dis. (2015) 61 (6): 859-863. doi:10.1093 /cid/civ633.

7. Dimitri Ceroni Georgios Kampouroglou Rebecca Anderson della Llana Davide Salvo. Osteoarticular infections in young children: ¿what has changed over the last years? Swiss Med Wkly. 2014;144:w13971. doi:10.4414/smw.2014.13971. 
8. Spellberg B Lipsky BA. Systemic antibiotic therapy for chronic osteomyelitis in adults. Clin Infect Dis. $2012 \mathrm{Feb}$ 1;54(3):393-407. doi: 10.1093/cid/cir842. Epub 2011 Dec 12.

9. Jason H. Calhoun M.D. F.A.C.S., M.M. Manring Ph.D. Mark Shirtliff Ph.D. Osteomyelitis of the long bones. Semin Plast Surg. 2009 May; 23(2): 5972.doi: $\underline{10.1055 / \mathrm{s}-0029-1214158 .}$

10. Jaspaul S. Gogia M.D. John P. Meehan M.D. Paul E. Di Cesare M.D. Amir A. Jamali M.D. Local Antibiotic Therapy in Osteomyelitis. Semin Plast Surg 2009;23:100-107. doi: 10.1055/s-0029$\underline{1214162\}}$

11. Charalampos G. Zalavras MD Randall E. Marcus MD L. Scott Levin MD Michael J. Patzakis MD. Management of Open Fractures and Subsequent Complications. J Bone Joint Surg Am, 2007 Apr; 89 (4): 884 -895. http://dx.doi.org/

12. Todd Jaeblon DO. Polymethylmethacrylate: Properties and Contemporary Uses in Orthopaedics. J Am Acad Orthop Surg 2010;18: 297-305.

13. Mauffrey $\mathrm{C}$ Hake ME Chadayammuri V Masquelet AC. Reconstruction of Long Bone Infections Using the Induced Membrane Technique: Tips and Tricks. Orthop Trauma. 2016 Jun;30(6):e188193. doi: $\underline{10.1097 / B O T .0000000000000500 . ~}$

14. Oosthuizen B Mole T Martin R Myburgh JG. Comparison of standard surgical debridement versus the VERSAJET Plus ${ }^{\mathrm{TM}}$ Hydrosurgery system in the treatment of open tibia fractures: a prospective open label randomized controlled trial. Int J Burns Trauma. 2014 Oct 26;4(2):53-58. eCollection 2014.

15. Melvin JS Dombroski DG Torbert JT Kovach SJ Esterhai JL Mehta S.
Open tibial shaft fractures: I. Evaluation and initial wound management. $\mathrm{J} \mathrm{Am}$ Acad Orthop Surg. 2010 Jan;18(1):1019.

16. Ludwig Labler MD Mario Rancan MD Ladislav Mica MD Luc Hårter PhD Daniela Mihic-Probst MD Marius Keel MD. Vacuum-Assisted Closure Therapy Increases Local Interleukin-8 and Vascular Endothelial Growth Factor Levels in Traumatic Wounds. Trauma. 2009; 66:749-757.

17. N. A. P. van Gestel J. Geurts D. J. W. Hulsen B. van Rietbergen S. Hofmann J. J. Arts. Clinical Applications of S53P4 Bioactive Glass in Bone Healing and Osteomyelitic Treatment: A Literature Review. BioMed Research International, vol. 2015, Article ID 684826, 12 pages, 2015. doi:10.1155/2015/684826

18. Alain C. Masquelet Thierry Begue. The Concept of Induced Membrane for Reconstruction of Long Bone Defects. Orthop Clin N Am 41 (2010) 27-37 doi:10.1016/j.ocl.2009.07.011.

19. Dale A Classen. Antibiotic bead pouch dressing. Winter 1996, Volume 4 Issue 4: 199-202

20. Cale Jacobs PhDChristian P. Christensen MD Michael E. Berend MD. Static and Mobile Antibiotic- impregnated Cement Spacers for the Management of Prosthetic Joint Infection. J Am Acad Orthop Surg 2009; 17: 356-368

21. Pang-Hsin Hsieh MD Kuo-Chin Huang MD Ching-Lung Tai PhD. Liquid Gentamicin in Bone Cement Spacers: In Vivo 
Antibiotic Release and Systemic Safety in Two-Stage Revision of Infected Hip Arthroplasty. J Trauma. 2009;66:804808. DOI: $\underline{10.1097 / T A .0 b 013 e 31818896 \mathrm{cc}}$

22. Seldes RM Winiarsky $\mathrm{R}$ Jordan LC Baldini $\mathrm{T}$ Brause B Zodda $\mathrm{F}$ et al. Liquid gentamicin in bone cement: a laboratory study of a potentially more costeffective cement spacer. J Bone Joint Surg Am. 2005;87:268-272. DOI: 10.2106/JBJS.C. 00728

23. Konstantinos N. Malizos Nikolaos E. Gougoulias Zoe H. Dailiana Sokratis Varitimidis Konstantinos A. Bargiotas Dionysios Paridis. Ankle and foot osteomyelitis: Treatment protocol and clinical results Injury. 2010 Mar;41(3):285293. doi: 10.1016/j.injury.2009.09.010.

24. Yong-Qing Xu Yue-Liang Zhu Xin-Yv Fan Tao Jin Yang Li Xiao-Qing He. Implant-Related Infection in the Tibia: Surgical Revision Strategy with Vancomycin Cement. ScientificWorldJournal. 2014; 2014: 124864. Published online 2014 Nov 5. doi: 10.1155/2014/124864

25. F. L. Game W J. Jeffcoate. Primarily non-surgical management of osteomielitis of the foot in diabetes. Diabetologia (2008) 51:962-967 DOI 10.1007/s00125008-0976

26. Sandra Saja Scherer M.D. Giorgio Pietramaggiori M.D. Jasmine C. Mathews M.A. Michael J. Prsa B.S. Sui Huang M.D. Ph.D Dennis P. Orgill M.D. Ph.D. The Mechanism of Action of the Vacuum-Assisted Closure Device. Plast Reconstr Surg. 2008 Sep;122(3):786797. doi: 10.1097/PRS.0b013e31818237ac.

27. Paul H. Kim MD Seth S. Leopold MD. Gustilo-Anderson Classification. Clin Orthop Relat Res (2012) 470:3270-3274 DOI 10.1007/s11999-012-23766.
28. DeCoster Thomas A. MD Bozorgnia Shahram MD. Surgical Techniques, Antibiotic Beads. Journal of the American Academy of Orthopaedic Surgeons: November 2008 - Volume 16 - Issue 11 - p $\underline{674-678}$

29. Cook GE Markel DC Ren W Webb LX McKee MD Schemitsch EH. Infection in Orthopaedics. J Orthop Trauma. 2015 Dec;29 Suppl 12:S19-23. doi: 10.1097/BOT.0000000000000461.

30. Carlos Pineda M.D. Rolando Espinosa M.D. Angelica Pena M.D. Radiographic Imaging in Osteomyelitis: The Role of Plain Radiography, Computed Tomography, Ultrasonography, Magnetic Resonance Imaging, and Scintigraphy. Semin Plast Surg 2009;23:80-89 . DOI $\underline{10.1055 / \mathrm{s}-0029-1214160}$

31. Yu Jin Lee Su Sadigh Kshitij Mankad Nikhil Kapse Gajan Rajeswaran.' The imaging of osteomielitis. Quant Imaging Med Surg 2016;6(2):184-198 . doi: $\underline{10.21037 / \text { qims.2016.04.01 }}$

32. Hatzenbuehler J Pulling TJ. Diagnosis and management of osteomyelitis. Am Fam Physician. 2011 Nov 1;84(9):10271033.

\section{CONFLICTO DE INTERES Y/O AGRADECIMIENTOS}

Los autores declaran que no existió ningún conflicto de interés en el presente reporte. 


\begin{tabular}{|l|l|}
\hline $\begin{array}{l}\text { Organismo más frecuente } \\
\text { en osteomielitis. }\end{array}$ & S. aureus. \\
\hline Cuerpo extraño asociado & $\begin{array}{l}\text { Staphylococcus coagulasa } \\
\text { negativo. }\end{array}$ \\
\hline $\begin{array}{l}\text { Mordidas, pie diabético y } \\
\text { úlceras de decúbito. }\end{array}$ & $\begin{array}{l}\text { Estreptococo y bacterias } \\
\text { anaerobias. }\end{array}$ \\
\hline $\begin{array}{l}\text { Enfermedad de células fal- } \\
\text { ciformes. }\end{array}$ & $\begin{array}{l}\text { Salmonella spp o Strepto- } \\
\text { coccus pneumaniae. }\end{array}$ \\
\hline $\begin{array}{l}\text { Pacientes inmunocompro- } \\
\text { metidos. }\end{array}$ & $\begin{array}{l}\text { Aspergillus spp, Candida } \\
\text { albicans o Mycobacteria } \\
\text { spp. }\end{array}$ \\
\hline VIH positivo. & $\begin{array}{l}\text { Bartonella henselae or B. } \\
\text { Quintana. }\end{array}$ \\
\hline
\end{tabular}

ANEXOS

Tabla 1. Relación clínica con el microorganismo

Fuente: propia 
microrganismos dentro del hueso, así como ocurre en el trauma o la cirugía.

Además, este es un sistema que no facilita para guiar ya sea el tipo de terapia antibiótica o quirúrgica.

Fuente: Propia

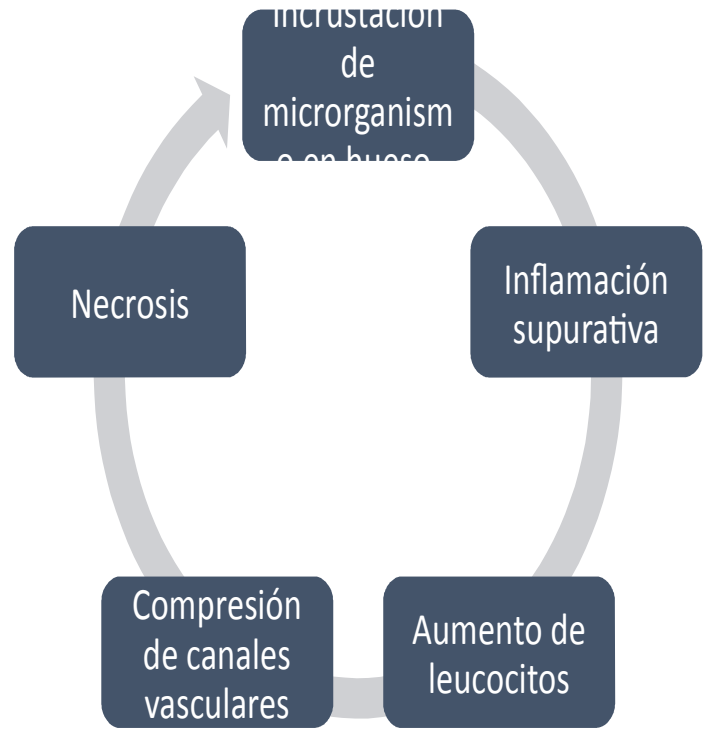

Figura 1. Mecanismo de la enfermedad Fuente: Porpia

Tabla 2. Sistema de Waldvogel

\begin{tabular}{l|l}
$\begin{array}{l}\text { Criterios de } \\
\text { clasificación }\end{array}$ & $\begin{array}{l}\text { *Duración del proceso infeccioso: } \\
\text { aguda o crónica. } \\
\text { *La fuente de la infección: } \\
\text { hematógena, por contigüidad } \\
\\
\text { Insuficiencia vascular. }\end{array}$
\end{tabular}

Limitaciones No considera el origen de la infección ya sea por penetrancia directa de 


\begin{tabular}{|l|l|}
\hline & compromiso local o sistémico. \\
\hline B & $\begin{array}{l}\text { El huésped presenta más de un } \\
\text { factor de compromiso local o } \\
\text { sistémico. }\end{array}$ \\
\hline C & $\begin{array}{l}\text { Pacientes severamente } \\
\text { comprometidos y el tratamiento } \\
\text { radical es necesario. }\end{array}$ \\
\hline
\end{tabular}

Fuente: Propia

Tabla 3. Sistema de Cierny-Mader

\begin{tabular}{|c|c|}
\hline \multicolumn{2}{|c|}{ Sistema de Cierny-Mader. } \\
\hline Estadio & Características \\
\hline $\begin{array}{c}\text { Estadio 1 } \\
\text { o estadio } \\
\text { medular. }\end{array}$ & $\begin{array}{c}\text { La osteomyelitis } \\
\text { Confinada a la cavidad medular } \\
\text { del hueso. }\end{array}$ \\
\hline $\begin{array}{c}\text { Estadio 2 } \\
\text { o estadio } \\
\text { superficial. }\end{array}$ & $\begin{array}{c}\text { La osteomielitis compromete la } \\
\text { cortical }\end{array}$ \\
& $\begin{array}{c}\text { Origen: principalmente } \\
\text { por inoculación directa o un } \\
\text { foco infeccioso contiguo. }\end{array}$ \\
\hline $\begin{array}{c}\text { Estadio 3 } \\
\text { o estadio } \\
\text { localizado. }\end{array}$ & $\begin{array}{c}\text { Compromiso de la cortical y } \\
\text { médula del hueso. } \\
\text { El hueso se mantiene estable. } \\
\text { El proceso infeccioso no } \\
\text { compromete la totalidad } \\
\text { del diámetro del hueso. }\end{array}$ \\
\hline $\begin{array}{c}\text { Estadio 4 } \\
\text { o estadio }\end{array}$ & $\begin{array}{c}\text { Compromete por completo el } \\
\text { grosor del hueso. }\end{array}$ \\
\hline
\end{tabular}

Fuente: Propia

Tabla 4. Subclasificación de CiernyMader

\section{Clasificació Características}

$\mathrm{n}$

A

El huésped no presenta 
Figura 3. Fractura de Tibia IIIB e inserción de cadenas o perlas de antibióticos seguida desbridación

Fuente: Properties and Contemporary Uses in Orthopaedics. J Am Acad Orthop Surg 2010;18: 297-305

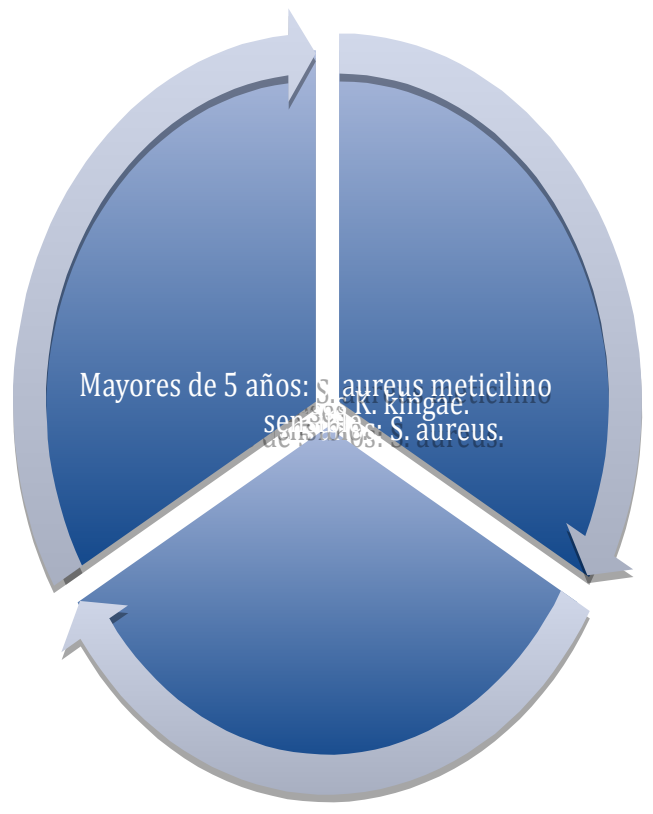

Figura 2. Etiología por grupos de edad

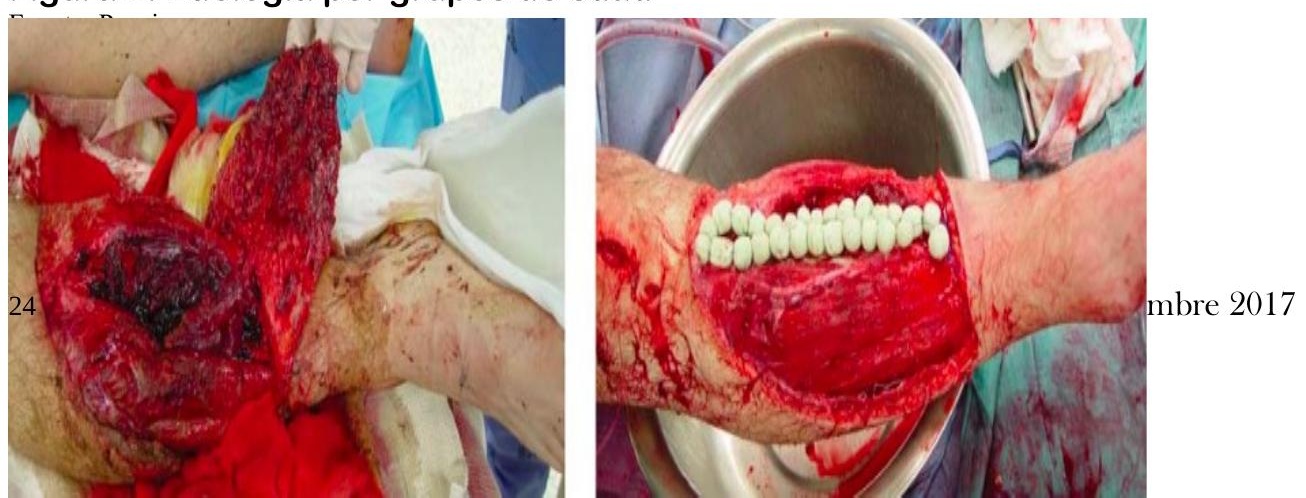


impregnated Cement Spacers for the Management of Prosthetic Joint Infection. J Am Acad Orthop Surg 2009;17: 356-368.
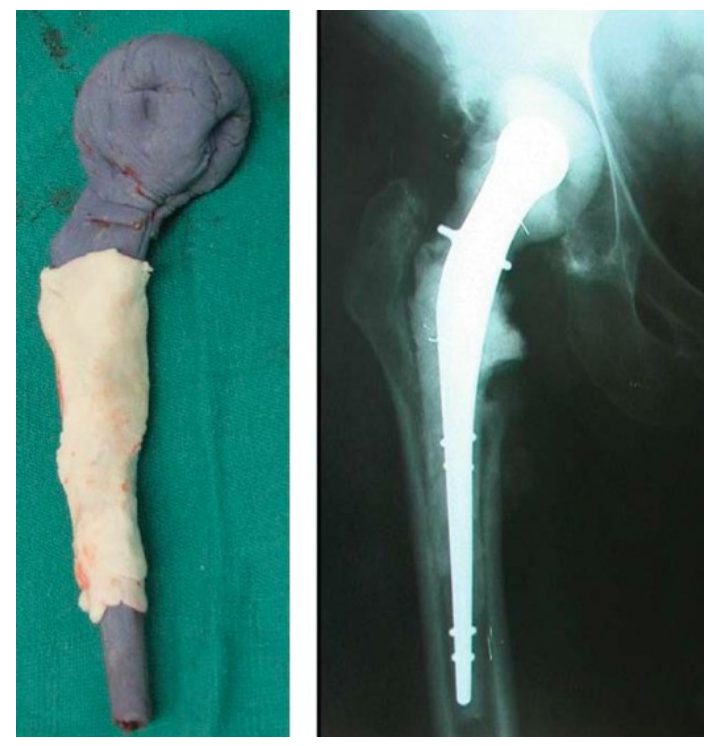

Figura 4. Modelo móvil de espaciador, con radiografía pos operatoria AP

Fuente: Cale Jacobs, PhDChristian P. Christensen, MD Michael E. Berend, MD. Static and Mobile Antibiotic- 